SPIE paper 2743-09

Aerospace/Defense Sensing and Controls

8-12 April 1996
Approved for public release

Distribution unlimited

\title{
Impact of display modulation transfer function on the quality of sampled imagery
}

\author{
Richard Vollmerhausen
}

U.S. Army CECOM Night Vision and Electronic

Sensors Directorate, Fort Belvoir, VA 22060

\begin{abstract}
This paper discusses the impact of display pixel shape, size and spacing on displayed image quality. The application of Fourier Transforms to sampled imagery is described. A characterization function for sampled imagers is derived by examining the image formation of a point source. Misapplication of the Sampling Theorem is also discussed.
\end{abstract}

Key words: sampled imagery, image quality, digital display, Sampling Theorem, transfer function, MTF, display pixel, sampling artifacts, Fourier Transform

\section{INTRODUCTION}

Many imaging systems have a modulation transfer function (MTF) such that the product of the transfer function times the Fourier Transform of the scene being imaged gives the Fourier Transform of the image being displayed. The transfer function quantifies how well the imaging system passes the spatial frequency content of the scene. We often judge the quality of an imaging system by looking at its MTF. Further, MTF is used as the independent variable in image quality and task metrics.

An MTF cannot be defined for imaging sensors which sample the scene. However, a response function can be defined which characterizes the performance of a sampled system in much the same way that MTF characterizes a non-sampled system. The response function consists of a transfer function term and a spurious response term. Both terms are important to the quality of sampled imagery and should be considered independently when establishing quality metrics.

This paper reviews the application of Fourier Transform theory to sampled systems. The transfer and spurious response terms for a sampled imager are derived. Misapplication of the Sampling Theorem is also discussed.

\section{CHARACTERIZING A SAMPLED IMAGER}

\subsection{Fourier Transform of a sampled image}

In a sampled imager, the scene content is sampled after filtering by the pre-sampling MTF of the sensor. If the sensor samples at spatial interval (X), then the Fourier Transform of the displayed image is: ${ }^{*}$

$$
I_{m g}(w) \underset{n \text { samples }}{=} \underset{i x}{f(n X)} P_{i x}(w) \exp ^{-j n w X}
$$

\footnotetext{
${ }^{\psi}$ Sensor horizontal and vertical response are assumed to be independent; the examples in this paper are one dimensional. 

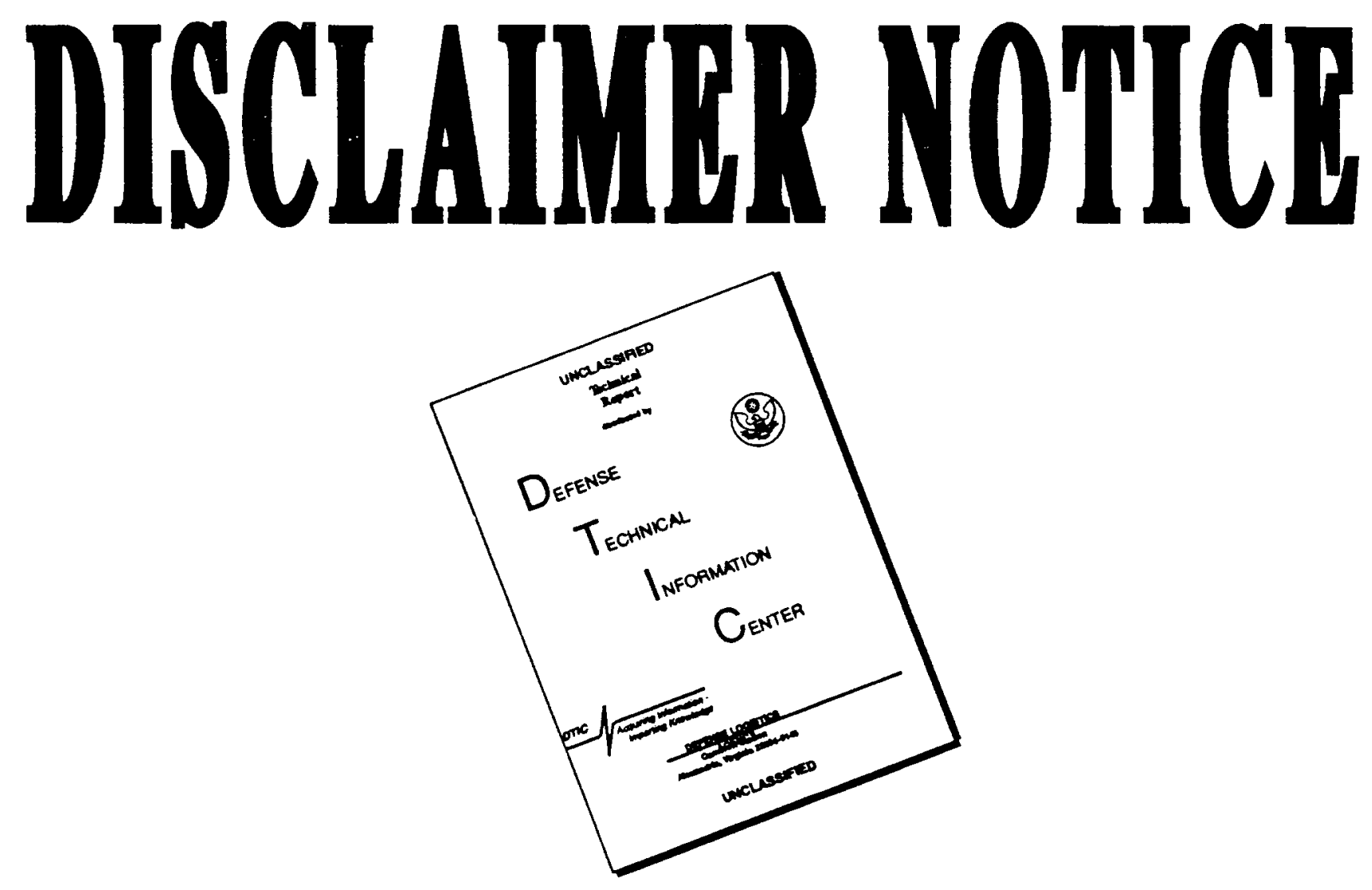

THIS DOCUMENT IS BEST

QUALITY AVAILABLE. THE COPY

FURNISHED TO DTIC CONTAINED

A SIGNIFICANT NUMBER OF

PAGES WHICH DO NOT

REPRODUCE LEGIBLY. 
where:

$$
\begin{aligned}
\mathrm{f}(\mathrm{x}) & =\text { sampled waveform } \\
& =\mathrm{h}(\mathrm{x}) \text { convolved with } \mathrm{s}_{\mathrm{cn}}(\mathrm{x}) \\
\mathrm{h}(\mathrm{x}) & =\text { pre-sample sensor point spread function } \\
\mathrm{s}_{\mathrm{cn}}(\mathrm{x}) & =\text { scene intensity distribution } \\
\mathrm{w} & =2 \pi \text { times spatial frequency } \\
\mathrm{H}(\mathrm{w}) & =\text { Fourier Transform of } \mathrm{h}(\mathrm{x}) \\
& =\text { pre-sampled MTF of sensor } \\
\mathrm{I}_{\mathrm{mg}}(\mathrm{w}) & =\text { Fourier Transform of image } \\
\mathrm{P}_{\mathrm{ix}}(\mathrm{w}) & =\text { Fourier Transform of pixel shape }
\end{aligned}
$$

The exponential places each display pixel at a sample position. The transform of the image is simply the sum of the transforms of the appropriately placed pixels.

Equation (1) allows the calculation of the image transform given the sample values and the pixel MTF. However, Equation (1) does not provide insight into the relationship between pre-sample MTF, sample spacing, and the display pixel MTF.

The following derivation follows a method often used to prove the Sampling Theorem. 1,2 The image can be constructed by convolving the pixel shape with delta functions at the sample points. The origin of the scene coordinate is not necessarily a sample point, so an offset (d) must be included for generality.

$$
\begin{aligned}
\mathrm{i}_{\mathrm{mg}}(\mathrm{x}) & =\sum_{\mathrm{n}} \int_{-\infty}^{\infty} \mathrm{f}\left(\mathrm{x}^{\prime}-\mathrm{d}\right) \delta\left(\mathrm{x}^{\prime}-\mathrm{nX}\right) \mathrm{p}_{\mathrm{ix}}\left(\mathrm{x}^{\prime}-\mathrm{x}\right) \mathrm{dx^{ \prime }} \\
& =\sum_{\mathrm{n}}\{\mathrm{f}(\mathrm{x}-\mathrm{d}) \delta(\mathrm{x}-\mathrm{nX})\}^{*} \mathrm{p}_{\mathrm{ix}}(\mathrm{x})
\end{aligned}
$$

where $\left({ }^{*}\right)$ means convolution and:

$$
\begin{aligned}
\mathrm{p}_{\mathrm{ix}}(\mathrm{x}) & =\text { pixel shape } \\
& =\text { Fourier Transform of } \mathrm{P}_{\mathrm{ix}}(\mathrm{w}) \\
\mathrm{i}_{\mathrm{mg}}(\mathrm{x}) & =\text { image intensity distribution } \\
& =\text { Fourier Transform of } \mathrm{I}_{\mathrm{mg}}(\mathrm{w})
\end{aligned}
$$

The Fourier Transform of $f(x)$ calculated in Equation (1) is for $f(x)$ windowed to the sampled interval. It does not matter in Equation (1) whether $\mathrm{f}(\mathrm{x})$ is actually limited to the sample interval or not. This is important in the new derivation because we need to define what we mean by $\mathrm{F}(\mathrm{w})$.

To accommodate the following derivation, we want to treat $f(x)$ as zero outside the sample interval. That is, the Fourier Transform of $\mathrm{f}(\mathrm{x})$ is the transform of the windowed function. This allows the substitution of an infinite set of samples in lieu of the finite set of samples; we now sample all space. Since convolution in space results in multiplication in frequency:

$$
\begin{aligned}
& I_{m g}(w)=\sum_{n=-\infty}^{\infty}\left[F(w) \exp ^{-j w d} * \delta(w-n u)\right] P_{i x}(w) \\
& I_{m g}(w)=\sum_{n=-\infty}^{\infty} F(w-n u) \exp ^{-j(w-n u) d} P_{i x}(w)
\end{aligned}
$$

where: $\quad u=2 \pi / X$ (the sample frequency in radians) 
Figure (1) is a notional plot of $\mathrm{I}_{\mathrm{mg}}(\mathrm{w})$. The image spectrum can be represented as $\mathrm{F}(\mathrm{w})$ replicated at multiples of $(\mathrm{u})$ and then weighted by $\mathrm{P}_{\mathrm{ix}}(\mathrm{w})$. Note that the phase of the replicas varies due to the $e^{-j(w-n u) d}$ multiplier. The image will vary with sample phase.

If Equation (2) is applied to a real sensor, then $s_{\mathrm{cn}}(\mathrm{x})$ is the scene limited by the sensor field of view. Also, $h(x)$, the point spread function, must be spatially limited. It is only under these circumstances that Equation (2) can substitute for Equation (1). However, a spatially limited function cannot be band limited. ${ }^{1}$ Aliasing is inevitable in a practical system. The replicas of $F(w)$ will overlap, preventing an exact reconstruction of the sampled signal. Of course, the effect of the aliasing can be very minor.

\subsection{Response function for a sampled imager}

In order to establish a characterizing response function $\mathrm{R}_{\mathrm{sp}}(\mathrm{w})$ for sampled imagers, we examine the image formation of a point source. 3,4 The function being sampled is the point spread function of the sensor, and the Fourier Transform of the point spread function is the sensor pre-sample MTF.

$$
\mathrm{R}_{\mathrm{Sp}}(\mathrm{w})=\sum_{\mathrm{n}=-\infty}^{\infty} \mathrm{H}(\mathrm{w}-\mathrm{nu}) \exp ^{-\mathrm{j}(\mathrm{w}-\mathrm{nu}) \mathrm{d}} \mathrm{P}_{\mathrm{iX}}(\mathrm{w})
$$

$\mathrm{R}_{\mathrm{sp}}$ (w) is symmetrical about zero frequency. Also, in practical systems, the display MTF will limit $\mathrm{R}_{\mathrm{sp}}(\mathrm{w})$ to the first two terms.

$$
\mathrm{R}_{\mathrm{sp}}(\mathrm{w})=\mathrm{H}(\mathrm{w}) \mathrm{P}_{\mathrm{ix}}(\mathrm{w})+\mathrm{H}(\mathrm{w}-\mathrm{u}) \exp ^{\mathrm{jud}} \mathrm{P}_{\mathrm{ix}}(\mathrm{w})
$$

The first term is exactly analogous to MTF for an analog system; it represents the transfer of scene information to the image. The second term represents spurious response introduced by the sampling and reconstruction. The phase factor common to both terms was dropped.

\subsection{Examples}

In order for the illustrations to be clear even after printing and reproduction, large pixels and gross sample spacing will be used in the examples. All of the examples are based on the picture of a wall clock shown in Figure (2). The picture was taken with a television camera. The horizontal and vertical MTF were measured and are shown in Figure (3).

In all of the following examples, the same display pixel size and shape are used but the sample spacing is changed. The square pixel is shown in the upper right corner of each picture. The transfer and spurious response calculations are done assuming a square pixel convolved with a narrow Gaussian to round the edges. The Gaussian dropped to $5 \%$ at an eighth the pixel width.

In Figure (4), the clock image is down-sampled by two in each direction. There are four sample positions for each pixel spacing in each direction resulting in sixteen samples per pixel area. The picture is well sampled, and the large pixels are well overlapped. The image is blurry but sampling artifacts are not apparent.

Figure (5) shows the calculated transfer and spurious responses for the image in Figure (4). Note that spatial frequency is in object space. Transfer response cuts off just above 0.2 cycles per milliradian and goes negative beyond that frequency. This result is expected; the pixel is square with a size (in object space) of 4.8 milliradians. Spurious response is almost zero everywhere. The spurious response is centered at the sample frequency of 0.83 cycles per milliradian and is therefore well filtered by the MTF of the large pixel. 
In Figure (6), the clock image is down-sampled by four in each direction. There are now two horizontal sample positions and two vertical sample positions for each pixel. The image is clearly interpretable, but sampling artifacts have appeared. Some edges are jagged, pixel intensities on the numbers are incorrect, and some of the minute markings are missing or out of place.

Figure (7) shows the calculated response for the sample spacing used in Figure (6). The transfer response has not changed. Now, however, the sampling frequency has been cut in half and the spurious response is centered at 0.42 cycles per milliradian. In this case, the display MTF cannot filter out the sampling artifacts.

The spurious response is in two parts. The response does not go to zero at zero frequency. The spurious response "reflects" off the amplitude axis; the phase changes because the imaginary component changes sign after reflection.

Figure (8) shows the clock image down-sampled eight times in both directions. Pixel size is the same as sample spacing. The sampling artifacts are quite bad. The seven, eight and nine numerals are illegible, and many of the minute markings are missing completely. Edges are very jagged. The image appears to be sharper because the pixels do not overlap.

Figure (9) shows the calculated transfer and spurious responses for the Figure (8) image. Again, transfer response is the same as in previous examples. Now, however, the spurious response is about the same as the transfer response.

The final example represents a practical problem which sometimes occurs in modern systems. The sensor is well sampled. However, the image is presented on a low resolution flat panel display or on a computer display using pixel replication to make the image larger. In this case, there is little aliasing, but the spurious response can still be very annoying.

Figure (10) shows an image of the numeral "3." Pixel size at the display is the same as in the previous examples. The image is magnified eight times because there is now one pixel for each of the original samples.

The transfer response is much better than in previous examples; see Figure (11). The pixel size in object space is only 0.6 milliradians. The small sample spacing (high sample rate) moves the spurious response away from the transfer response; they do not overlap. However, the high pixel MTF results in a visible spurious response. In this case, the spurious response can, of course, be removed by proper display techniques. ${ }^{4}$

\section{MISAPPLICATION OF THE SAMPLING THEOREM}

The Sampling Theorem is often used to provide a "yes/no" answer to the complicated question of whether a sensor and associated display are adequately sampled. The Sampling Theorem is actually of little value in evaluating real sensor and display systems.

The Sampling Theorem does not quantify either the penalty of under-sampling or the benefits of one display choice over another. Further, the often quoted "two samples per cycle" criteria represents a mathematical, idealized extreme; this criteria is not useful with real sensors and displays.

Nothing in this discussion should be construed as suggesting that sampling at a rate double the effective MTF cutoff is inadequate. For most sensors, sampling at a frequency double the point where MTF reaches ten to forty percent is generally adequate for decent image quality. 


\subsection{Description of the Sampling Theorem}

The Sampling Theorem states that, for a signal $\mathrm{f}(\mathrm{x})$ for which the Fourier Transform has no components above frequency $\frac{Z}{\mathrm{f}_{\text {samp }}}$ inclusive, the function can be entirely reconstructed by the series:

$$
\mathrm{f}(\mathrm{x})=\sum_{\mathrm{n}=-\infty}^{\infty} \mathrm{f}\left(\mathrm{n} / \mathrm{f}_{\text {samp }}\right) \sin \left(\pi \mathrm{f}_{\text {samp }} \mathrm{x}-\mathrm{n} \pi\right) /\left(\pi \mathrm{f}_{\text {samp }} \mathrm{x}-\mathrm{n} \pi\right)
$$

A band-limited function can be uniquely determined from its values at a sequence of equidistant points, $1 / \mathrm{f}_{\text {samp }}$ apart. The above series is used to reconstruct the function. Each term in the above series is a sample function $[\sin (\mathrm{x}) /(\mathrm{x})]$, also referred to as a sinc wave. The sinc wave amplitude is equal to the sample value, and the period is such that the sinc wave crosses zero at all other sample points. The function is sampled over all space, and each sinc wave extends in both directions over all space.

\subsection{Example}

Figure (12) shows ten cycles of a sine wave; Figure (13) is the Fourier Transform. Taking the first zero as the "band-limit," the function is sampled 2.2 times per cycle. The asterisks in Figure (12) show the sample points.

Figure (14) shows one of the sinc waves generated to reconstruct the sampled function. Figure (15) shows the result of sampling and reconstructing one period near the middle. Finally, sinc waves are generated for all 22 samples and added together. The resulting reconstruction is shown in Figure (16).

The sixth cycle is poorly sampled due to the sample phase. It reproduces well in the final result because it is surrounded by other sampled periods of the sine wave. The edges are poorly sampled also but, having neighbors on only one side, they do not reconstruct to the full amplitude.

Note that the Sampling Theorem does not suggest that a single cycle of a sine wave can be reconstructed by taking two samples. The Fourier Transform of a single cycle of the sine wave is shown in Figure (17). Using the first zero as the "band-limit," the sample rate would be 4.0 per cycle. If the sine wave were infinitely extended, then the Fourier Transform would be a delta function at the sine wave frequency, and just over two samples per cycle would be adequate.

\subsection{Discussion}

Complete knowledge of a band-limited function over a local interval defines the function over all space. ${ }^{1}$ The converse is also true: the local interval can be constructed by sampling at remote locations. The Sampling Theorem uses this property of a band-limited function. When using 2.2 samples per cycle in the above example, a single cycle of the sine wave was not reconstructed by sampling only that cycle. The middle of the function was reconstructed by sampling the whole function.

Information about a local area of an image does not reside at remote locations in the image. We try to engineer the point spread function of a sensor to be finite, hopefully rather small. Also, spatially discrete pixels are used on the display. The tenets of the Sampling Theorem do not apply.

Further, even if the conditions specified in the Sampling Theorem were constructed, and certainly reasonable approximations are achievable, the Sampling Theorem does not suggest that a single cycle of a sine wave can be reconstructed by taking two samples. The "two samples per cycle" criteria, so often applied when evaluating real sensors, is a misapplication of the Sampling Theorem. 


\section{CONCLUSIONS}

Fourier analysis does apply to sampled imagers and can be used to find a response function analogous to the MTF of analog systems. The characterizing function for a sampled imager is found by examining the image formation of a point source. Sampled systems have a transfer response identical to the MTF of analog systems. However, the spurious response resulting from the combination of sample spacing, presample MTF and display pixel shape is also important. With sampled imagers, image quality and task metrics become a multivariate problem, with the transfer and spurious response of the imager treated independently.

The "two samples per cycle" criteria based on the Sampling Theorem represents an idealized limit. The "two sample" criteria does not apply to real sensors and displays. Further, the Sampling Theorem cannot be used to quantify either the penalty of under-sampling or the benefits of one display choice over another.

\section{REFERENCES}

1. J.M. Wozencraft and I.M. Jacobs, Principles of Communication Engineering, Appendix 8A and Appendix 5B, John Wiley \& Sons, New York, 1965.

2. R.N. Bracewell, The Fourier Transform and Its Applications, Chapter 10, McGraw-Hill Book Company, New York, 1986.

3. W. Wittenstein, J.C. Fontanella, A.R. Newbery, and J. Baars, "The definition of the OTF and the measurement of aliasing for sampled imaging systems," Optica Acta, Vol. 29, No. 1, pp 41-50, 1982.

4. R. Vollmerhausen, "Display of Sampled Imagery," IRIA-IRIS Proceedings: Meeting of the IRIS Specialty Group on Passive Sensors, Vol. 1, pp 175-192, 1990. 


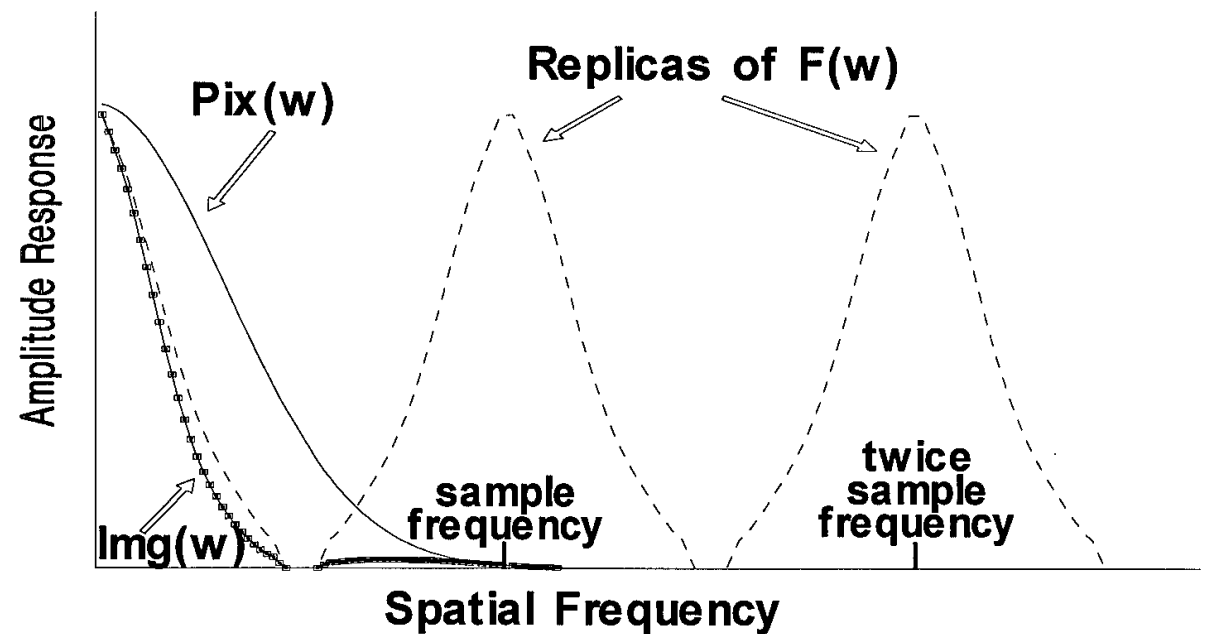

Figure 1. Notional plot of sampled spectrum, display MTF, and resulting image.

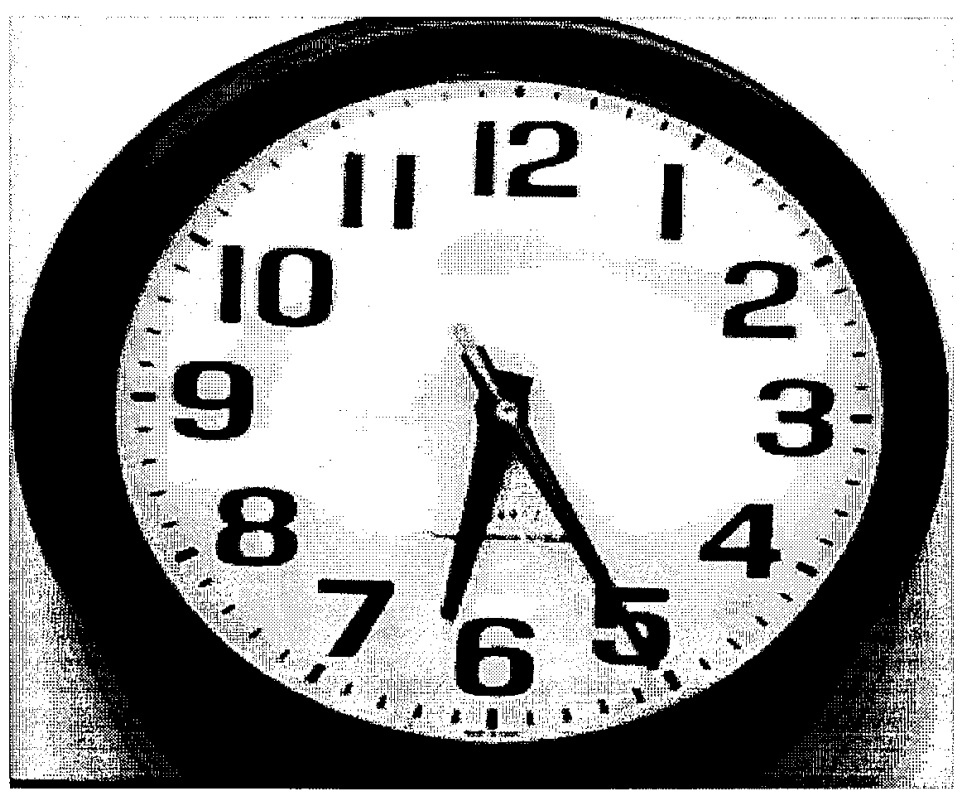

Figure 2. Picture of wall clock.

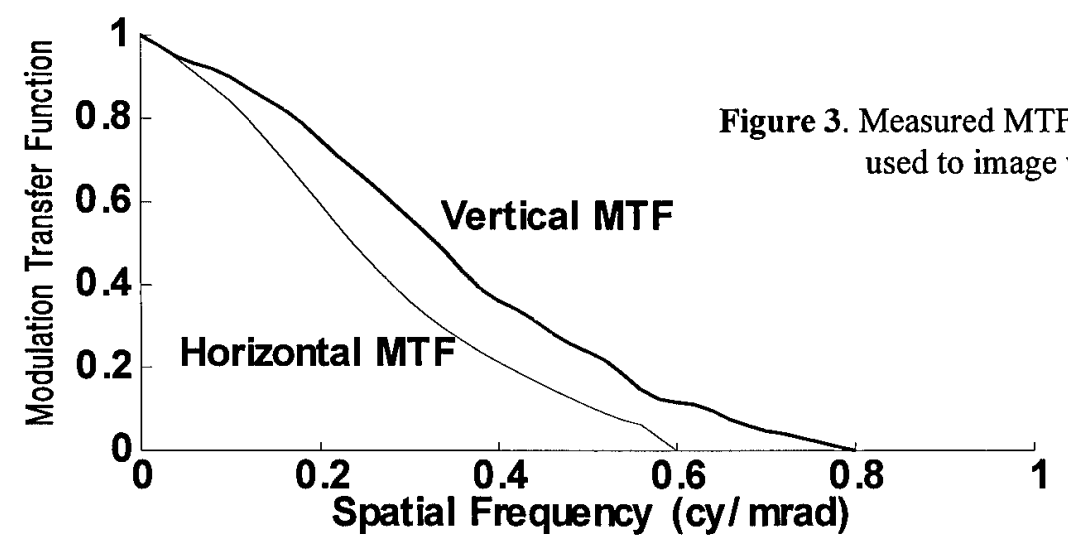




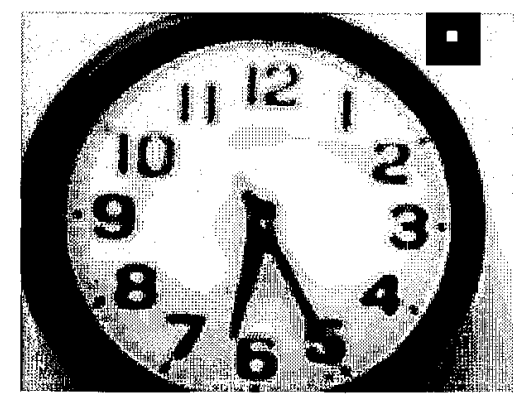

Figure 4. Clock down- sampled by two in each direction. Four samples per display pixel each direction.

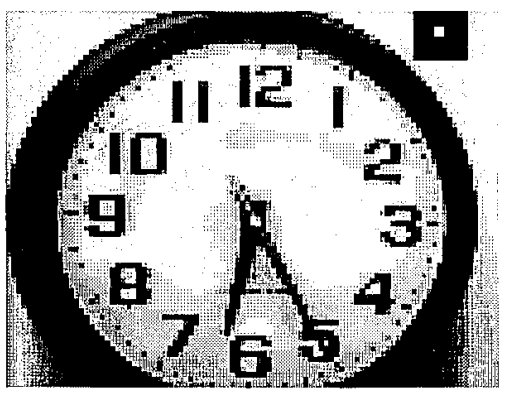

Figure 6. Clock down-sampled by four in each direction. Two samples per display pixel in each direction.

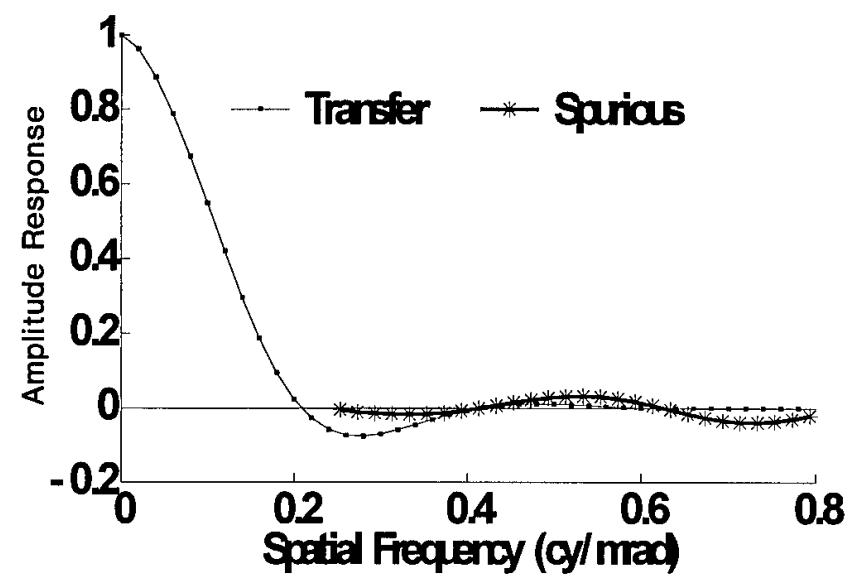

Figure 5a. Horizontal transfer and spurious response for sampling in Figure 4.

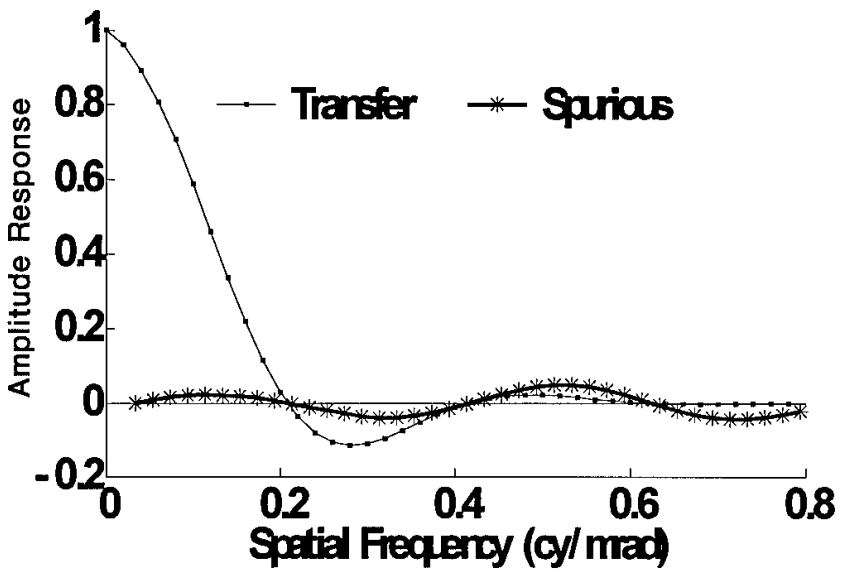

Figure 5b. Vertical transfer and spurious response for sampling in Figure 4.

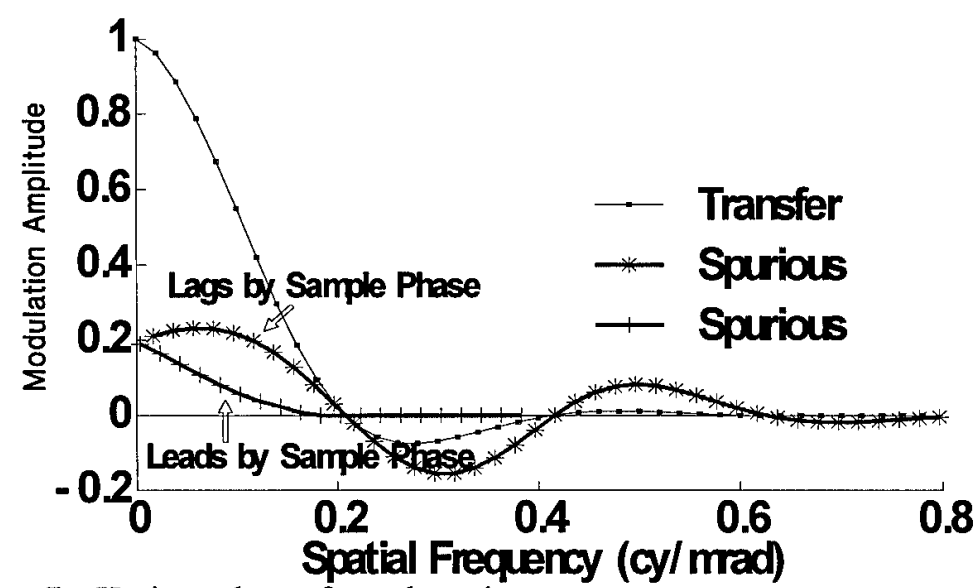

Figure 7a. Horizontal transfer and spurious response for sample spacing of Figure 6. 


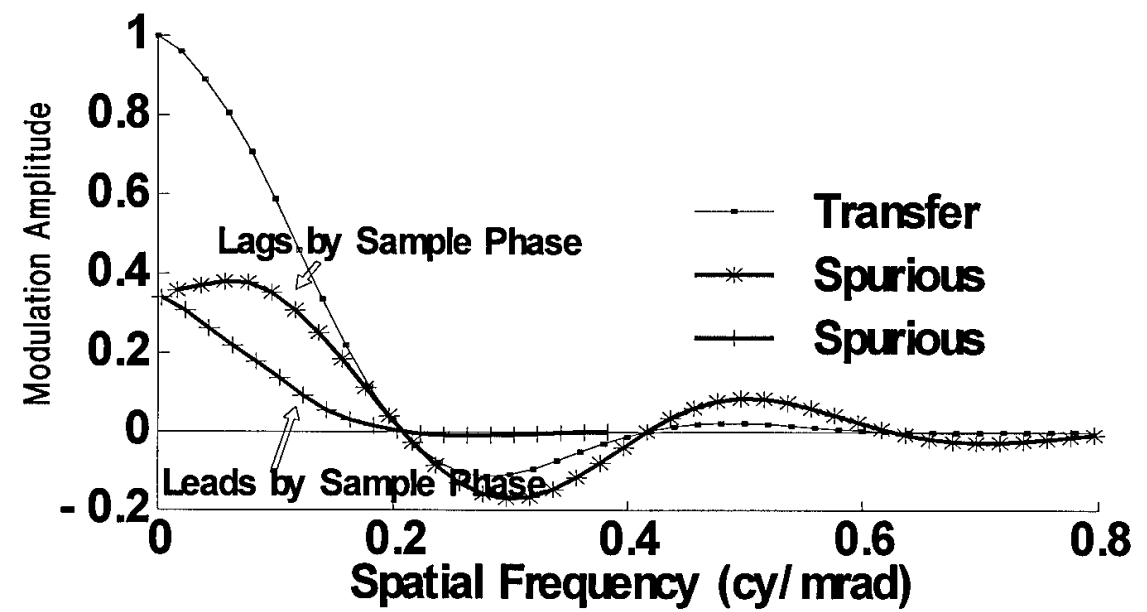

Figure $7 \mathbf{b}$. Vertical transfer and spurious response for sampling in Figure 6.

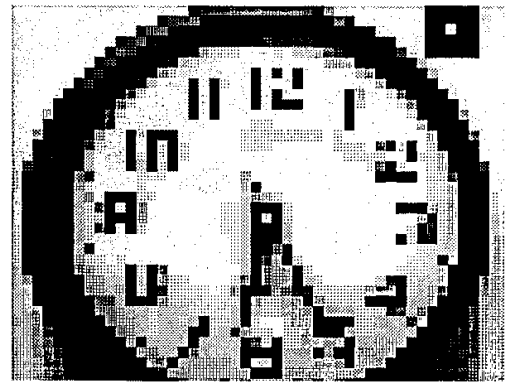

Figure 8. Clock down-sampled by eight in each direction. One sample per pixel.

Figure 9b. Vertical transfer and spurious response for sampling shown in Figure 8.

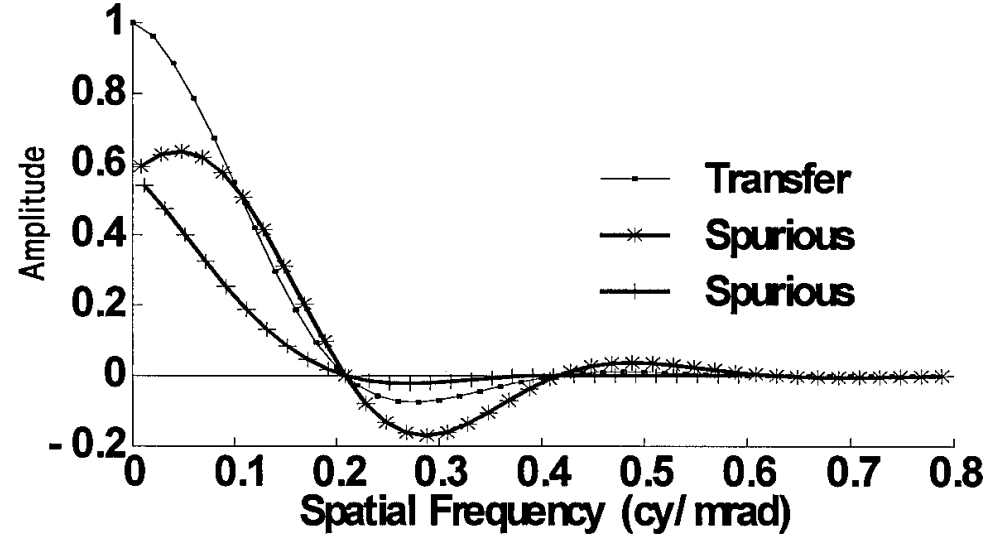

Figure 9a. Horizontal transfer and spurious response for sampling shown in Figure 8.

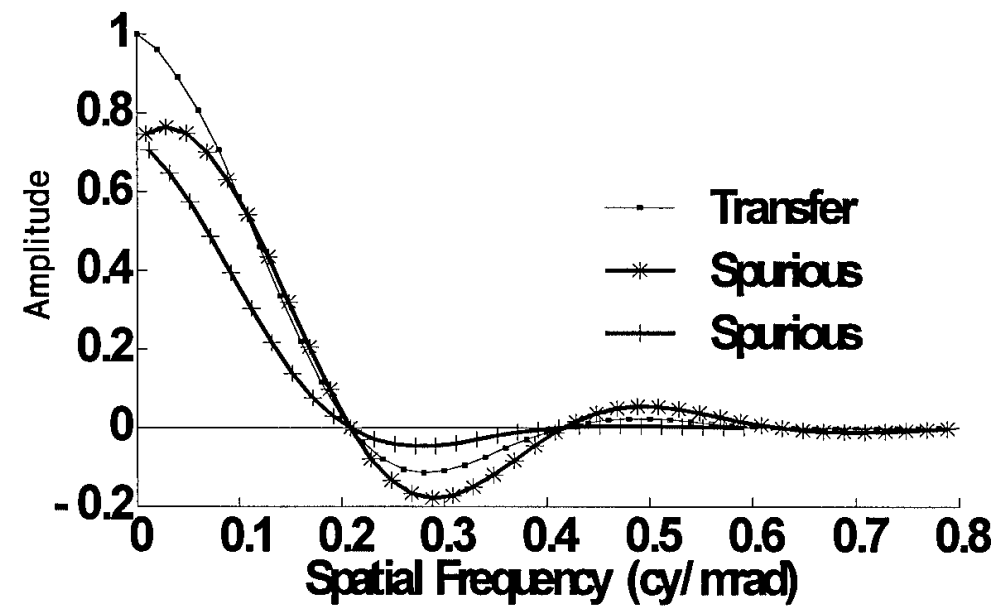




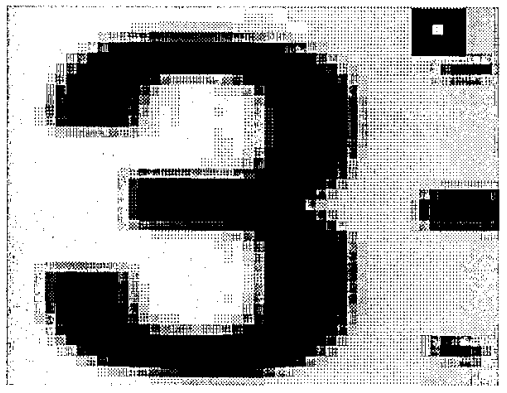

Figure 10. Numeral " 3 " of clock picture. Each original sample is one display pixel.

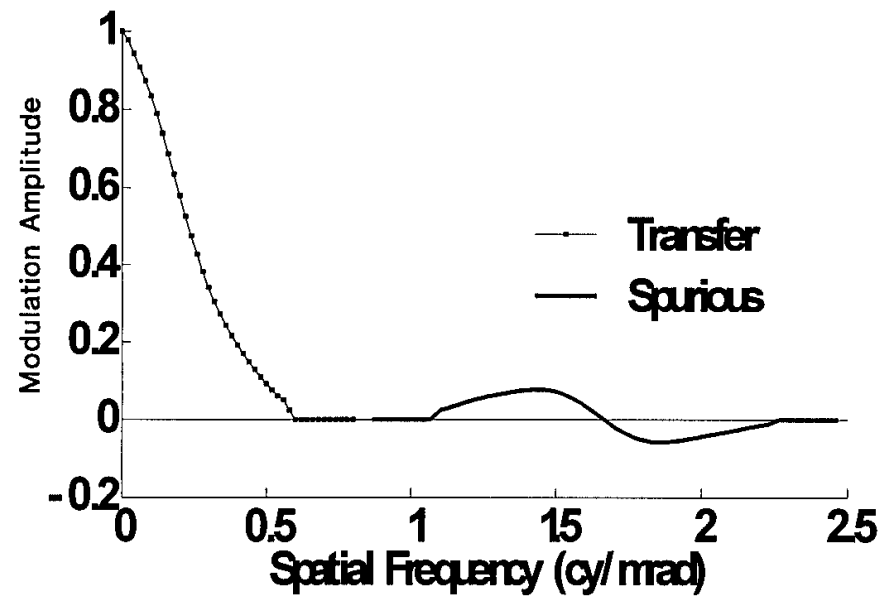

Figure 11a. Horizontal transfer and spurious response for Figure 10.

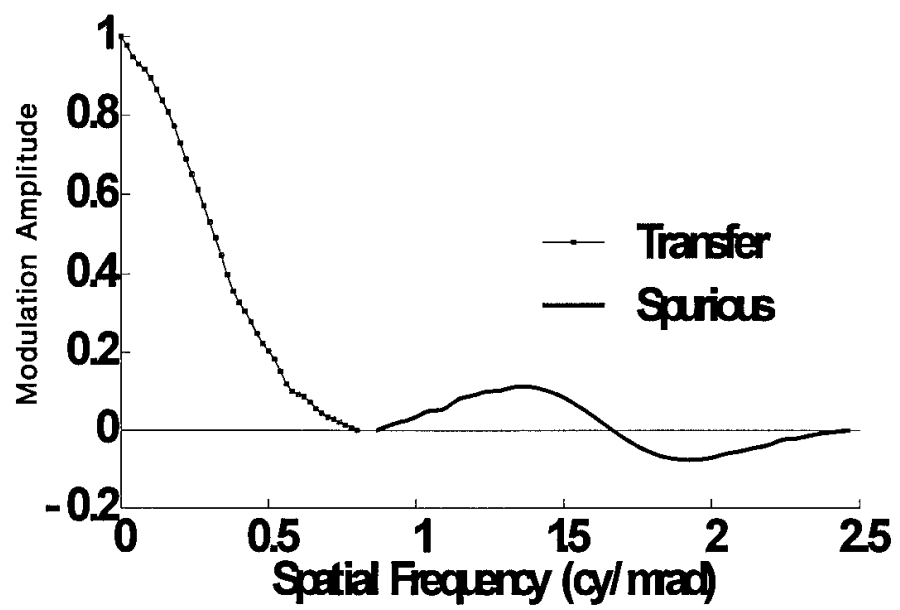

Figure 11b. Vertical transfer and spurious response for Figure 10.

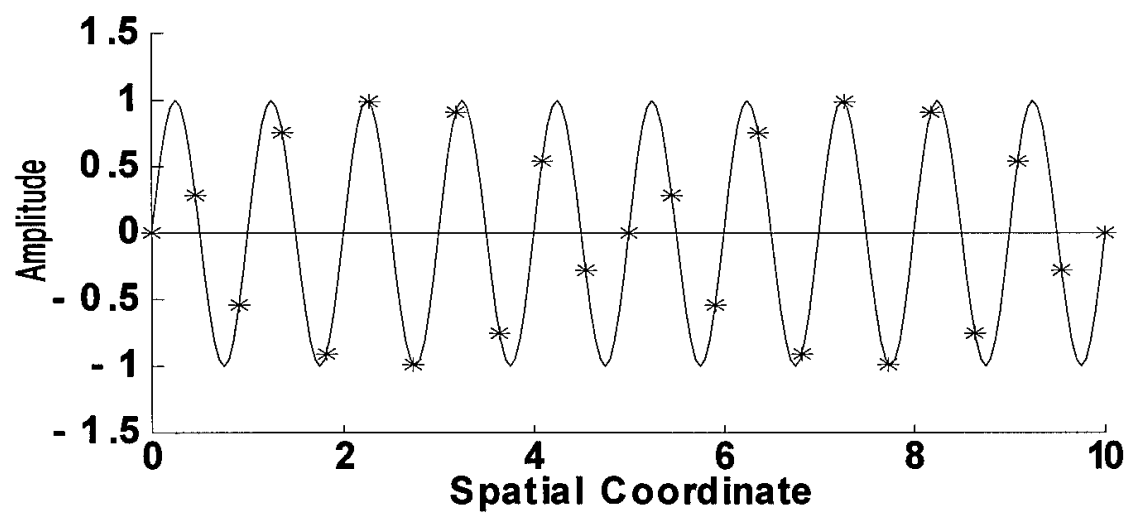

Figure 12. Ten periods of a sine wave sampled 2.2 times per cycle at the $\left(^{*}\right)$. 


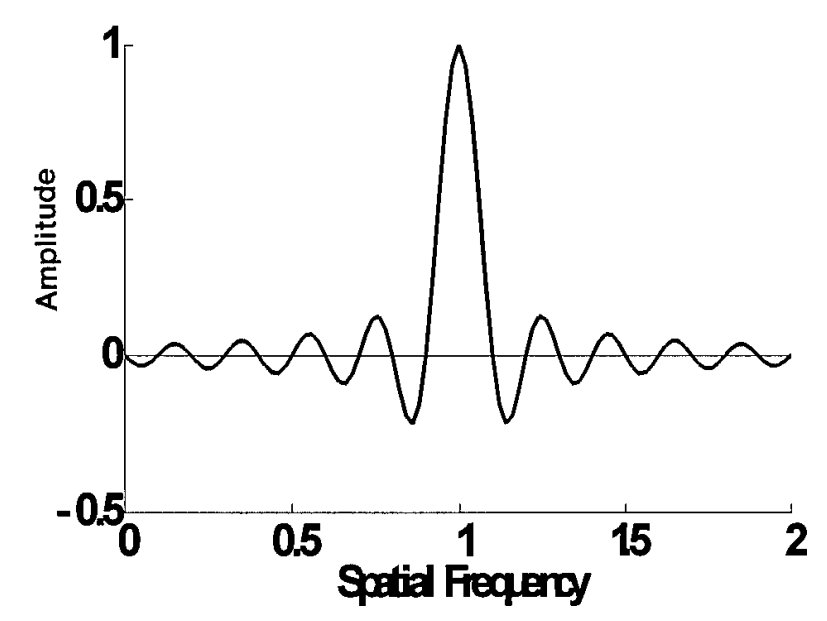

Figure 13. Fourier Transform of sine waves in Figure 12.

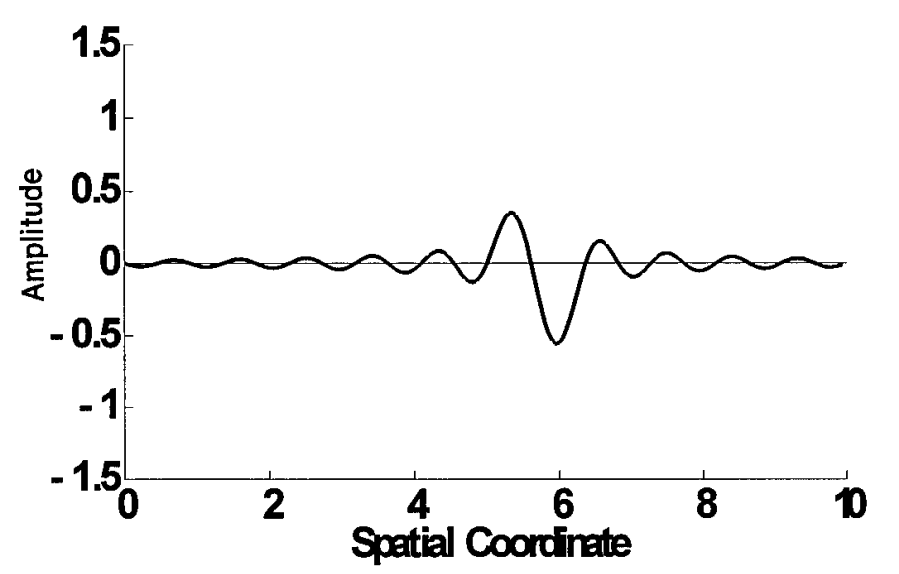

Figure 15. Reconstruction using the two samples of the 6 th cycle.

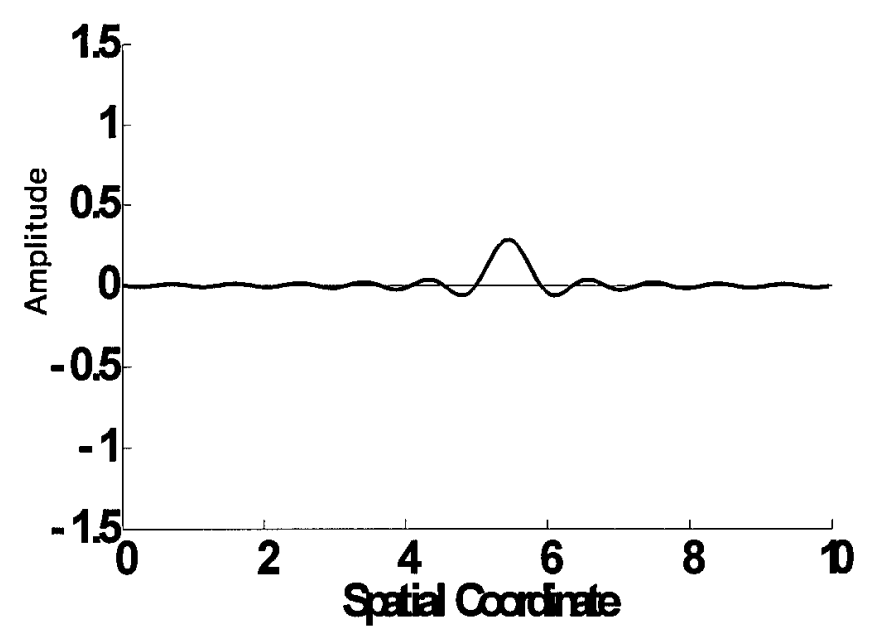

Figure 14. One sinc wave of the Sampling Theorem reconstruction.

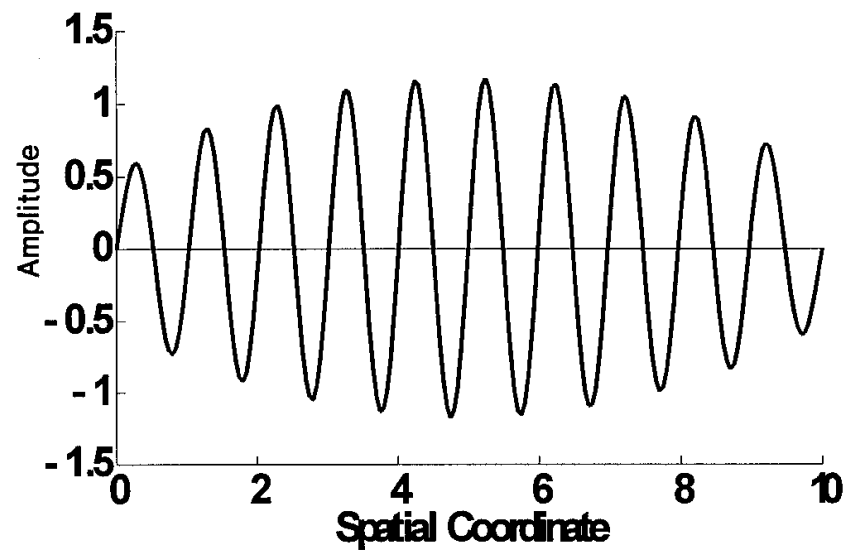

Figure 16. Reconstruction using all 22 samples of the ten cycles.

Figure 17. Frequency spectrum of one cycle of a sine wave with period of one.

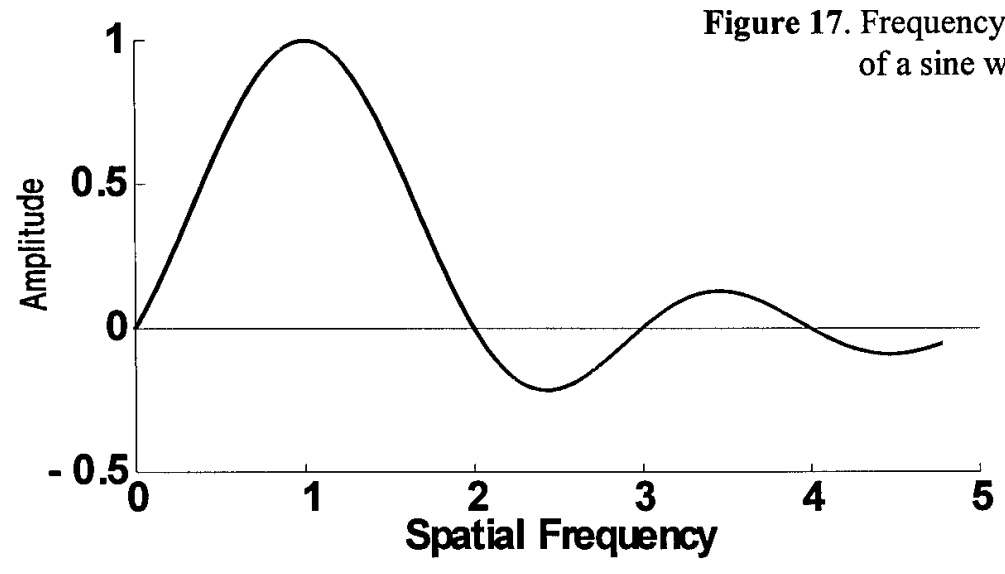

